\title{
Android Application to Convert Speech to Text and Text to Speech
}

\author{
Manish $\mathbf{Y M}^{1}$, Kavya $\mathrm{K}^{2}$, Vishesh $\mathrm{S}^{3}$ \\ Student, Department of ISE, BNMIT, Bangalore, India ${ }^{1,2}$ \\ BE, Department of TCE, VTU, Belgaum, India ${ }^{3}$
}

\begin{abstract}
: $21^{\text {st }}$ century is the age of smartphones [1] and other smart gadgets like tablets, portable media players, pocket computers, and smart watches. Most of these gadgets run android OS developed by Google and Open Handset Alliance (OHA) [2]. Android OS supports many android applications, either developed by the company itself or by any third party called third party application (app). In this paper, we have presented one of the applications developed by us which can perform the task of converting speech to text and text to speech. The speech of the subject will be carefully and intelligently converted to text by formal approximations. Anything written or copied to the clipboard can be converted to speech. Our goal is to operate using English language. This can be later employed in the case of regional languages as well.
\end{abstract}

Keywords: third party application (app), converting speech to text, converting text to speech, recording the data entered on to the clipboard, recording the speech at different rate and publishing it in suitable format.

\section{INTRODUCTION}

Smartphones support the traditional data movement and Telephony, along with many futuristic and smart applications. Many of the applications are developed by native developers of the OS and come built-in with the smartphone. The major advantage of $21^{\text {st }}$ century smartphones running on a particular OS- it supports third party applications. Any developer can design, develop and publish an application of his choice and is made available in the market or store. There are huge variety of apps, including video games, music products and business tools available on store. [3]

\section{Problem Statement}

An android application is capable of

- converting speech to text

- converting text to speech

- recording the data entered on to the clipboard

- recording the speech at different rate and publishing it in suitable format

\section{SOFTWARE REQUIREMENTS SPECIFICATION}

A. Functional Requirements

Functional requirements define the functionality of a system to be developed

- A clipboard/ text space to enter the data to be converted to speech

- Access to microphone of the device on touch

- Intelligent approximation of the pronounced speech

- Easy access to storage media of the device

\section{B. Non-functional requirements}

Non-functional requirements play the behaviour and performance of the system at its critical stages

- Provision has to be made to accommodate loads of data

- A buffer to enrich processing at critical stages

- Security of the data /media is of great importance

\section{APPLICATION LAYOUT}

A. Home screen

Application consists of a home screen, by selecting suitable options one can navigate to desired screens having specific functionalities. Figure 1 shows the home screen of the application. 
Vol. 8, Issue 2, February 2019

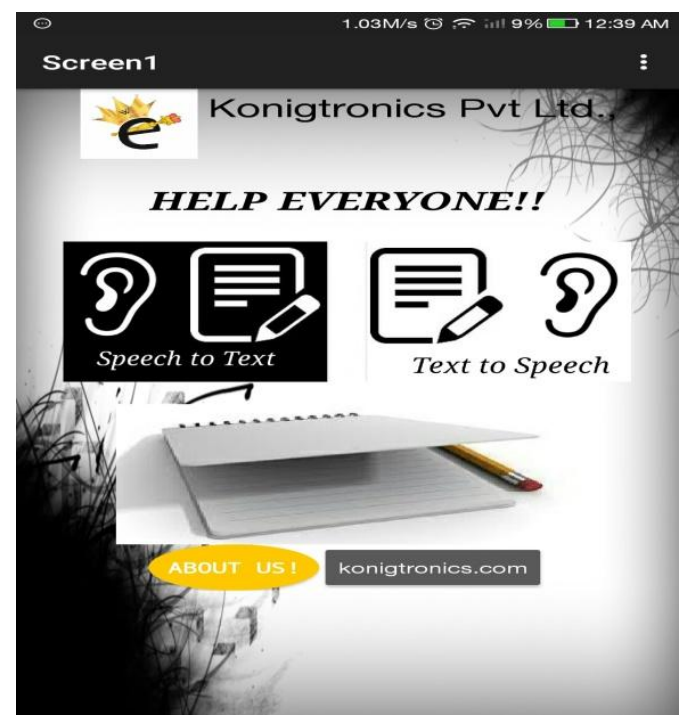

Figure 1 shows the home screen of the application.

B. Speech to text converter

By selecting the speech to text converter tab on the home screen one can navigate to the speech to text converter screen. Here a 'speak' button starts recording the speech of a person by enabling the microphone of the smartphone. Figure 2 shows the speech to text converter screen.

C. Text to speech converter

By selecting the text to speech converter tab on the home screen one can navigate to the text to speech converter screen. Clipboard/ Text board allows the subject to enter the text to be read out loud by the computer using Artificial Intelligence. The frequency of the speech can also be controlled. Figure 3 shows the text to speech converter screen.

D. Other components

We have employed various other components like hyperlink, SD card storage option and share button to allow storage and communication of the recorded data /voice. Background images, thumbnails, icons and graphical information play an important role in beautifying the application. Figure 4 shows a canvas screen and option to store the pattern to the device.

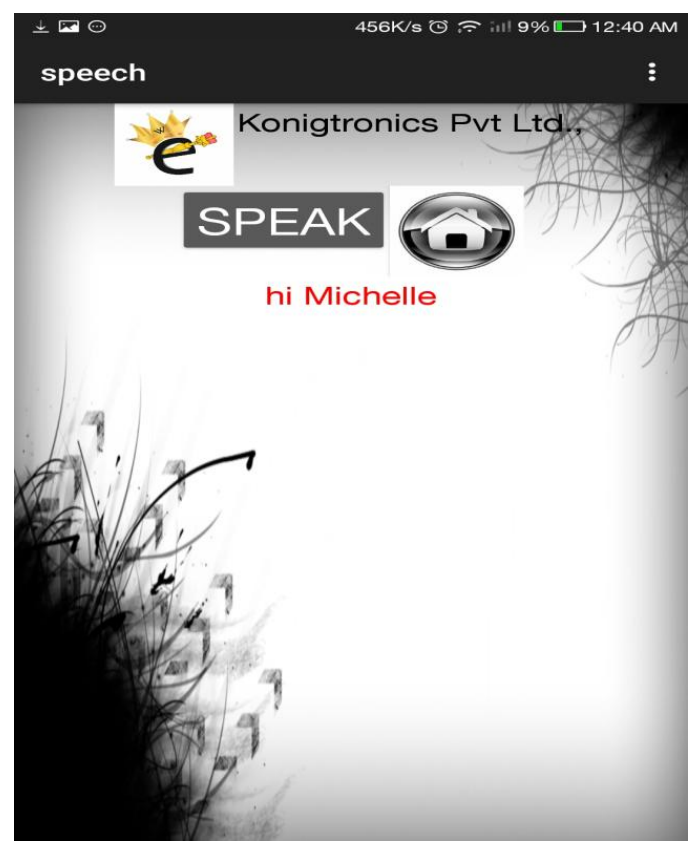

Figure 2 shows the speech to text converter screen. 
Vol. 8, Issue 2, February 2019

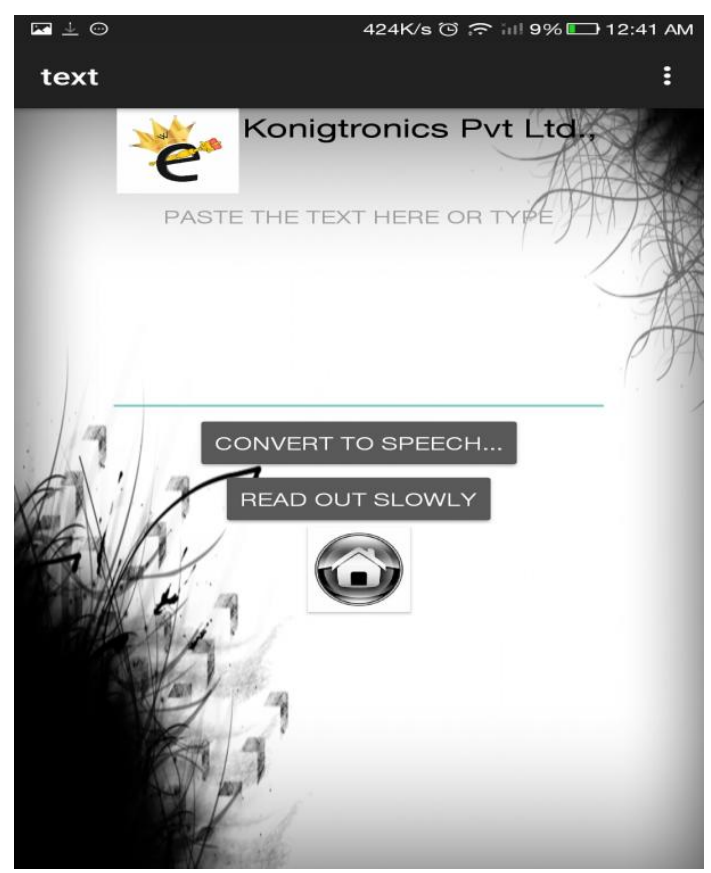

Figure 3 shows the text to speech converter screen.

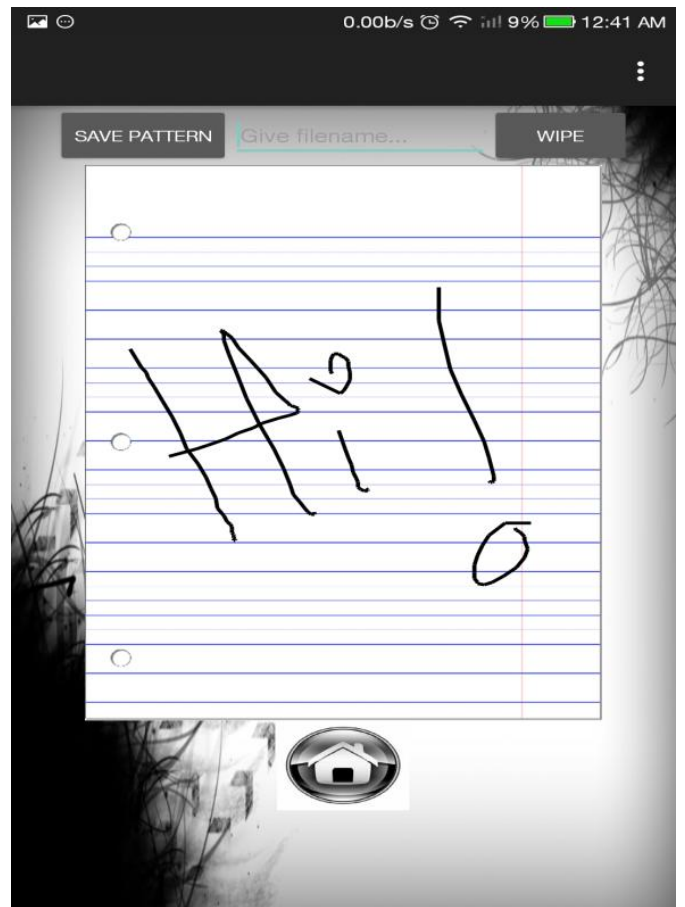

Figure 4 shows a canvas screen and option to store the pattern to the device.

\section{CONCLUSIONS}

Intelligent and smart systems have the ability to perform the task which human fail to perform or unable to perform. A person who is mute cannot spread its thought through speech. A person who is deaf fails to receive information spread through word of mouth. This application plays an important role in converting the speech to text and vice versa, thereby acting as a life support to the above. Both functionalities in one app are a boon to the user. 
Vol. 8, Issue 2, February 2019

\section{REFERENCES}

[1] Smartphone usage in the 21st century: who is active on WhatsApp? Christian Montag 1,3*, Konrad Błaszkiewicz 2 , Rayna Sariyska 1, Bernd Lachmann1, Ionut Andone 2, Boris Trendafilov 2, Mark Eibes 2 and Alexander Markowetz 2- Montag et al. BMC Res Notes (2015) 8:331 DOI 10.1186/s 13104-015-1280-z

[2] Research on Android app Vs. Apple app Market: Who is leading? Shivam Jaiswal 1, Ajay Kumar 2- International Journal of Engineering and Computer Science ISSN: 2319-7242 Volume 3 Issue 4 April, 2014 Page No. 5553-5556

[3] Getting started - Android Apps- https://ilc.com.au/wp-content/uploads/2015/01/Getting-Started-Apps-for-Android.pdf

\section{OUR GUIDE}

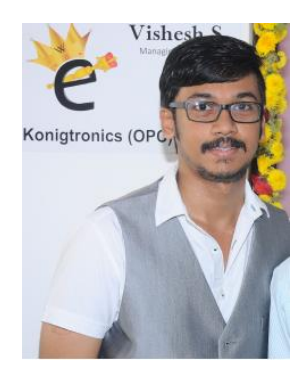

VISHESH $\mathbf{S}$ born on $13^{\text {th }}$ June 1992, hails from Bangalore (Karnataka) and has completed B.E in Telecommunication Engineering from VTU, Belgaum, Karnataka in 2015. He also worked as an intern under Dr. Shivananju BN, former Research Scholar, Department of Instrumentation, IISc, Bangalore. His research interests include Embedded Systems, Wireless Communication, BAN and Medical Electronics. He is also the Founder and Managing Director of the corporate company Konigtronics Private Limited. He has guided over a hundred students/interns/professionals in their research work and projects. He is also the co-author of many International Research Papers. He is currently pursuing his MBA in e-Business and PG Diploma in International Business. Presently Konigtronics Private Limited has extended its services in the field of Software Engineering and Webpage Designing. Konigtronics also conducts technical and non-technical workshops on various topics. 